\title{
Odd Viscosity in the Quantum Critical Region of a Holographic Weyl Semimetal
}

\author{
Karl Landsteiner, ${ }^{1, *}$ Yan Liu, ${ }^{2,1,3, \dagger}$ and Ya-Wen Sun ${ }^{1, \$}$ \\ ${ }^{1}$ Instituto de Física Teórica UAM/CSIC, C/Nicolás Cabrera 13-15, Universidad Autónoma de Madrid, \\ Cantoblanco, 28049 Madrid, Spain \\ ${ }^{2}$ School of Space and Environment, Beihang University, Beijing 100191, China \\ ${ }^{3}$ School of Physics, Huazhong University of Science and Technology, Wuhan 430074, China
}

(Received 23 April 2016; published 19 August 2016)

\begin{abstract}
We study odd viscosity in a holographic model of a Weyl semimetal. The model is characterized by a quantum phase transition from a topological semimetal to a trivial semimetal state. Since the model is axisymmetric in three spatial dimensions there are two independent odd viscosities. Both odd viscosity coefficients are nonvanishing in the quantum critical region and nonzero only due to the mixed axial gravitational anomaly. It is therefore a novel example in which the mixed axial gravitational anomaly gives rise to a transport coefficient at first order in derivatives at finite temperature. In the quantum critical region, the physics of viscosities as well as conductivities is governed by the quantum critical point.
\end{abstract}

DOI: 10.1103/PhysRevLett.117.081604

One of the most surprising outcomes of string theory is the application of the AdS/CFT correspondence to the physics of strongly interacting quantum many-body systems [1]. The need to develop models that allow us to address the question of real-time transport in strongly interacting quantum fluids has arisen from experiments in completely different areas of physics: in the quark gluon plasma generated in heavy ion collisions, the collective behavior of ultracold atoms, the strange metal phase of the high- $T_{c}$ superconductors, and most recently the hydrodynamic electronic flow observed in graphene and similar materials [2-5].

Graphene is a "Dirac" semimetal in which the electrons are well described by the Dirac equation. The motion of electrons in graphene is, however, restricted to two spatial dimensions. In the last few years, new materials whose electronics is described by the Dirac or Weyl equation in three spatial dimensions have been demonstrated [6-11]. These Weyl semimetals have a plethora of exciting and exotic transport properties related to the chiral anomaly of three dimensional relativistic fermions.

As in graphene the electron fluid within a Weyl semimetal might as well be strongly interacting due to the smallness of the Fermi velocity compared to the speed of light. It seems therefore natural to ask if holography can be applied to such systems as well. In this case holography should play a similar important role for the understanding of quantum transport of Weyl semimetals as it already does in the theory of the quark gluon plasma [12]. In particular we ask the question if one can learn something new from holographic models utilizing universal properties of these materials such as the (effective) presence of chiral anomalies. We will address this question and answer it to the affirmative.
Recently a holographic model of a Weyl semimetal has been developed in $[13,14]$. Let us briefly review the most salient feature of this model. Its action is given by (1) with $\mathcal{F}_{\mu \nu}=\partial_{\mu} V_{\nu}-\partial_{\nu} V_{\mu}, F_{\mu \nu}=\partial_{\mu} A_{\nu}-\partial_{\nu} A_{\mu}$, and $D_{\mu} \Phi=\left(\partial_{\mu}-i q A_{\mu}\right) \Phi$. The holographic dictionary determines the field content of the model. The metric encodes the dynamics of the energy momentum tensor.

$$
\begin{aligned}
S= & \int d^{5} x \sqrt{-g}\left\{\frac{1}{2 \kappa^{2}}\left(R+\frac{12}{L^{2}}\right)-\frac{1}{4 e^{2}} \mathcal{F}^{2}-\frac{1}{4 e^{2}} F^{2}\right. \\
& -\left(D_{\mu} \Phi\right)^{*}\left(D^{\mu} \Phi\right)-V(\Phi) \\
& \left.+\epsilon^{\mu \nu \rho \sigma \tau} A_{\mu}\left[\frac{\alpha}{3}\left(3 \mathcal{F}_{\nu \rho} \mathcal{F}_{\sigma \tau}+F_{\nu \rho} F_{\sigma \tau}\right)+\zeta R_{\delta \nu \rho}^{\beta} R_{\beta \sigma \tau}^{\delta}\right]\right\} .
\end{aligned}
$$

There are two gauge fields. The first one, denoted by $V_{\mu}$, is dual to a conserved vector $U(1)$ current that can be identified with the electric current. The second one, $A_{\mu}$, is an axial gauge field. It couples to the complex scalar field $\Phi$ via an axial covariant derivative. The axial current suffers also from the axial anomaly which has three parts: one is the electromagnetic contribution to the axial anomaly, the second one is the purely axial $U(1)_{A}^{3}$ anomaly, and the third one is the gravitational contribution to the axial anomaly (i.e., mixed axial gravitational anomaly). These three anomalies are represented by the Chern-Simons terms in the action (1). The scalar field potential is chosen to be $V(\Phi)=m^{2}|\Phi|^{2}+\frac{\lambda}{2}|\Phi|^{4}$. The mass determines the dimension of the operator dual to $\Phi$ and we chose it to be $m^{2} L^{2}=$ -3 [15]. The boundary value of the scalar field is dual to a mass deformation in the field theory. 
In [14] the boundary conditions [16]

$$
\lim _{r \rightarrow \infty} r \Phi=M, \quad \lim _{r \rightarrow \infty} A_{z}=b,
$$

together with asymptotic AdS behavior of the metric were considered. Choosing, furthermore, the scalar field charge $q=1$ and the scalar self-coupling $\lambda=1 / 10$ it was found that the model undergoes a quantum phase transition as a function of the dimensionless parameter $M / b$. Note that the mixed axial gravitational anomaly is included in the holographic Weyl semimetal model (1) while it does not play any role in all the discussions of [14], including the phase transition and electric conductivities. This model can be understood as a gravity analogue of the Lorentz breaking Dirac system with Lagrangian

$$
\left[\gamma^{\mu}\left(i \partial_{\mu}-e v_{\mu}-\gamma_{5} b \delta_{\mu}^{z}\right)+M\right] \Psi=0 .
$$

This Lorentz breaking Dirac system has been used as a model for Weyl semimetals before in, e.g., [19-22].

At zero temperature for $M / b<0.744$ the scalar field vanishes in the IR towards $r=0$ whereas the axial gauge field takes a nonvanishing value $\left.A_{z}\right|_{r=0}=b_{\text {eff }}$. In this regime the model has a nonvanishing anomalous Hall conductivity given by $\sigma_{\mathrm{AHE}}=8 \alpha b_{\text {eff }}$. For $M / b>0.744$ the axial gauge field vanishes in the IR whereas the scalar field takes a finite value that is determined by the minimum of the potential $V^{\prime}(\Phi)=0$. In this phase the anomalous Hall conductivity vanishes. The model undergoes therefore a topological quantum phase transition between a topological semimetal state with nonvanishing anomalous Hall conductivity and a trivial semimetal state with vanishing anomalous Hall conductivity. There is an emergent Lifshitz symmetry at the critical point $M / b \simeq 0.744$ and it governs the quantum critical physics at finite temperature [23]. An illustration for our model (1) is shown in Fig. 1.

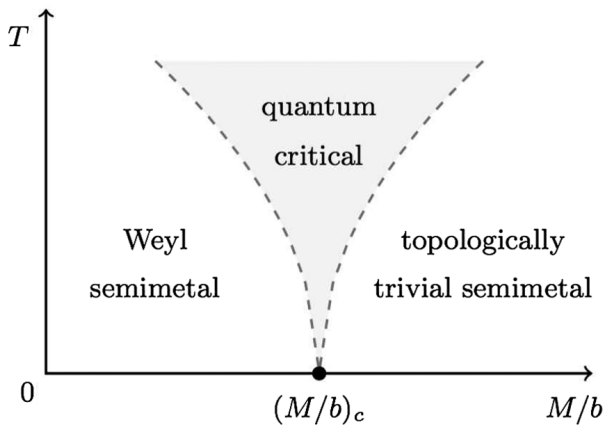

FIG. 1. The schematic picture for the holographic Weyl semimetal model (1) in the coupling constant $(M / b)$-temperature ( $T$ ) plane. At zero temperature a topological quantum phase transition occurs at the critical value $(M / b)_{c}$. At finite temperature the dashed line is a smooth crossover and in the quantum critical regime the physics is governed by the quantum critical behavior.
A necessary ingredient for the presence of odd viscosity is broken time reversal symmetry [24-27]. This is in principle provided by the axial gauge field background $b$. We note, however, that this is a UV parameter and we could expect that the viscosity is determined rather by the IR properties, similar to the anomalous Hall conductivity. It follows then that in the topological trivial phase in which time reversal symmetry is restored at the end point of the holographic RG flow $b_{\text {eff }}=0$ we should not expect substantial odd viscosity. On the other hand one can also argue that odd viscosity should be absent in the topological phase at zero temperature. At weak coupling the argument goes as follows: the low energy effective model describing a Weyl semimetal is

$$
S=\int d^{4} x \bar{\Psi}\left(i \gamma^{\mu} \partial_{\mu}-e \gamma^{\mu} v_{\mu}-\gamma_{5} \gamma_{z} b_{\text {eff }}\right) \Psi .
$$

By a field redefinition the parameter $b_{\text {eff }}$ can be removed from the action at the cost of introducing the anomalous effective term

$$
\begin{aligned}
\Gamma_{\text {anom }}= & \int d^{4} x \sqrt{-\gamma}\left(b_{\text {eff }} z\right) \epsilon^{\mu \nu \rho \lambda}\left(\alpha \mathcal{F}_{\mu \nu} \mathcal{F}_{\rho \lambda}+\frac{\alpha}{3} F_{\mu \nu} F_{\rho \lambda}\right. \\
& \left.+\zeta R^{\alpha}{ }_{\beta \mu \nu} R^{\beta}{ }_{\alpha \rho \lambda}\right) .
\end{aligned}
$$

The anomaly (5) encodes the response at zero temperature and shows that there is Hall conductivity but no odd (Hall) viscosity. Rather, the gravitational response is third order in derivatives as the Riemann curvature is second order in derivatives on the metric. We note that at finite temperature this derivative counting is not necessarily correct anymore. A well-known example for this is the contribution of the mixed axial gravitational anomaly to the chiral vortical effect [28-31]. As we will show now in our holographic model the gravitational contribution to the axial anomaly is also able to induce odd viscosity (a first order effect in derivatives) once temperature is switched on.

In an axisymmetric system characterized by a time reversal breaking vector such as $\vec{b}$ there are seven [32] independent viscosities [26]. We are interested in the two independent odd viscosity tensor components. Generically, we can define the viscosities via the Kubo formula

$$
\eta_{i j, k l}=\lim _{\omega \rightarrow 0} \frac{1}{\omega} \operatorname{Im}\left[G_{i j, k l}^{R}(\omega, 0)\right],
$$

with the retarded Green's function of the energy momentum tensor

$G_{i j, k l}^{R}(\omega, 0)=-\int d t d^{3} x e^{i \omega t} \theta(t)\left\langle\left[T_{i j}(t, \vec{x}), T_{k l}(0,0)\right]\right\rangle$. 
We chose our coordinates such that $\vec{b}=b \hat{e}_{z}$. The two odd viscosities related to the antisymmetric part of the retarded Green's function under the exchange of $(i j) \leftrightarrow(k l)$ are ( $T$ denotes the index combination $x x-y y$ )

$$
\eta_{H_{\|}}=-\eta_{x z, y z}=\eta_{y z, x z}, \quad \eta_{H_{\perp}}=\eta_{x y, T}=-\eta_{T, x y} .
$$

In this sector there are also two even (or shear) viscosities $\eta_{\|}=\eta_{x z, x z}=\eta_{y z, y z}$ and $\eta_{\perp}=\eta_{x y, x y}=\eta_{T, T}$.

We note that $\eta_{H_{\perp}}$ can be understood as Hall viscosity in the plane orthogonal to $\vec{b}$ whereas $\eta_{H_{\|}}$is specific to axisymmetric three dimensional systems. The later has been shown to arise also via the coupling of elastic gauge fields to the electron gas in Weyl semimetals [33]. In that case the odd or Hall viscosity is best thought of as a property of the phonon gas arising via the electron-phonon Chern-Simons interactions. This effective Hall viscosity is related to the underlying Hall conductivity of the electron gas and arises from the electronic point of view as an axial Hall conductivity. In contrast here we will be dealing with Hall or odd viscosity as an intrinsic property of the strongly coupled electron fluid. Hall viscosity arising from gravitational $\theta$ terms in holographic models dual to $2+1$ dimensional field theories has been studied before in, e.g., [34-40]. In contrast our system is dual to a $3+1$ dimensional theory with a mixed axial gravitational anomaly represented by the five dimensional gravitational Chern-Simons term in (1).

We use the following ansatz for the background at finite temperature

$$
\begin{gathered}
d s^{2}=-u d t^{2}+\frac{d r^{2}}{u}+f\left(d x^{2}+d y^{2}\right)+h d z^{2}, \\
A=A_{z} d z, \quad \Phi=\phi,
\end{gathered}
$$

with the fields $u, f, h, A_{z}, \phi$ real functions of $r$. It turns out that they are independent of the Chern-Simons couplings $\alpha$ and $\zeta$ and the numerical solutions have been studied in [14]. At finite temperature there is a horizon at a finite value $r=r_{0}$. The entropy density is given by the area element of the horizon $s=\left.4 \pi f \sqrt{h}\right|_{r=r_{0}}$. In order to probe the interesting nontrivial IR physics and relate our findings to possible applications to physical Weyl semimetals we should work at small temperatures. At higher temperatures it is rather the UV completion of the model that is probed.

Longitudinal viscosity.-In order to compute the viscosities we switch on the following perturbations: $\delta g_{i z}=h_{i z}(r) e^{-i \omega t}, \delta A_{i}=a_{i}(r) e^{-i \omega t}$ for $i \in\{x, y\}$. They form the complex combination $h_{ \pm}=h_{x z} \pm i h_{y z}$ and $a_{ \pm}=a_{x} \pm i a_{y}$. The solutions to lowest order in $\omega$ can be written as [41]

$$
\begin{aligned}
h_{ \pm}= & r^{2}-\frac{M^{2}}{3}+\frac{M^{4}(2+3 \lambda)}{18} \frac{\ln r}{r^{2}} \\
& +\frac{1}{r^{2}}\left[f_{3}+\frac{\omega}{4}\left(\left.\left[i \frac{f^{2}}{\sqrt{h}} \pm 4 \zeta \frac{q^{2} A_{z} \phi^{2} f^{2}}{h}\right]\right|_{r=r_{0}}\right)\right]+\ldots
\end{aligned}
$$

near the conformal boundary. Here $f_{3}$ is the coefficient of the $1 / r^{2}$ term in the asymptotic expansion of metric function (see [42]). From the first order term in $\omega$ we can read off the odd and even viscosity coefficients. The dissipationless odd viscosity is

$$
\eta_{H_{\|}}=\left.4 \zeta \frac{q^{2} A_{z} \phi^{2} f^{2}}{h}\right|_{r=r_{0}},
$$

whereas the dissipative even viscosity is $\eta_{H_{\|}}=$ $\left.\left(f^{2} / \sqrt{h}\right)\right|_{r=r_{0}}$. The dissipative viscosity is a form of shear viscosity. Here we only want not note that it has a nontrivial dependence on $M / b$ and can drop significantly below the KSS bound as has been noted before for anisotropc holographic systems (for more details see the Supplemental Material [42]).

The dissipativeless odd viscosity $\eta_{H_{\|}}$as a function of $M / b$ for small but finite temperatures is shown in Fig. 2 . It is highly suppressed in the Weyl semimetal part of the phase diagram but rises steeply as the quantum critical region around $M / b \simeq 0.744$ is entered. It peaks roughly at the critical value and then falls off in a somewhat slower fashion as $M / b$ increases. The extreme $M / b \rightarrow \infty$ limit can be reached by setting $b=0$ keeping $M$ finite. In this case the field $A_{z}$ is simply zero along the holographic RG flow and from (11) it follows that the odd viscosity vanishes again in this limit.

Transverse viscosity.-We switch on the perturbations $\delta g_{x x}-\delta g_{y y}=2 h_{L}(r) e^{-i \omega t}, \delta g_{x y}=h_{x y}(r) e^{-i \omega t}$ and form the complex combination $H_{ \pm}=h_{L} \pm i h_{x y}$. Expanding to first order in $\omega$ we find

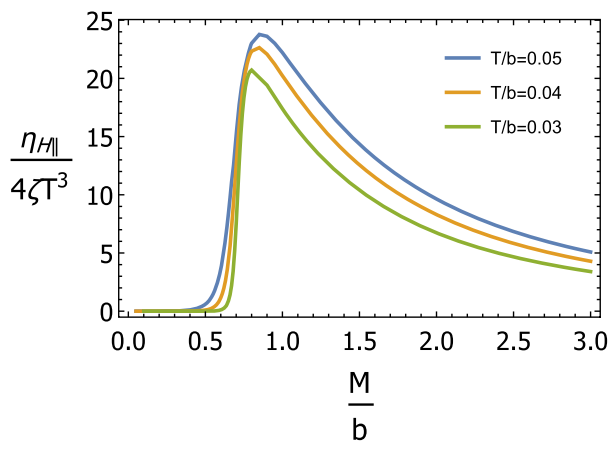

FIG. 2. Odd viscosity $\eta_{H_{\|}}$as a function of $M / b$ at different temperatures. 


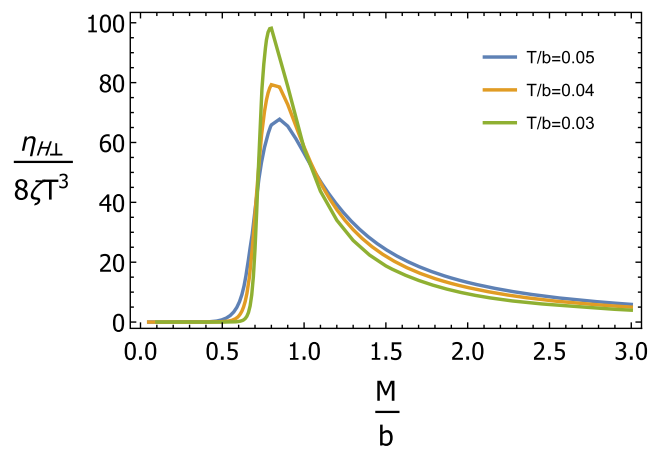

FIG. 3. Odd viscosity $\eta_{H_{\perp}}$ as a function of $M / b$ at different low temperatures.

$$
\begin{aligned}
H_{ \pm}= & r^{2}-\frac{M^{2}}{3}+\frac{M^{4}(2+3 \lambda)}{18} \frac{\ln r}{r^{2}} \\
& +\frac{1}{r^{2}}\left[f_{3}+\frac{\omega}{4}\left(\left.\left[\text { if } \sqrt{h} \pm 8 \zeta q^{2} \phi^{2} f A_{z}\right]\right|_{r=r_{0}}\right)\right]+\cdots
\end{aligned}
$$

near the conformal boundary. Using the holographic dictionary we can read off the viscosities from the terms at first order in $\omega$ at order $1 / r^{2}$ in the large $r$ expansion. The nondissipative odd viscosity is

$$
\eta_{H_{\perp}}=\left.8 \zeta q^{2} \phi^{2} f A_{z}\right|_{r=r_{0}} .
$$

The even shear viscosity in this sector obeys the usual holographic bound $4 \pi \eta_{\perp}=s$. Again, we find that in the low temperature regime the nondissipative odd viscosity has substantial support only in the quantum critical region around $M / b \simeq 0.744$ as can be seen from Fig. 3. On the other hand, in the regime in which the horizon probes the UV completion of our holographic model such that $r_{0} \rightarrow \infty$ (the extreme high temperature regime) we find $2 \eta_{H_{\|}}=\eta_{H_{\perp}}=8 \zeta q^{2} M^{2} b$. In this high temperature regime there is no trace left of the quantum phase transition at zero temperature.

We also note that the analytic results on the viscosities (together with our previous result on the conductivities in [14]) allow us to obtain the nontrivial relation

$$
\frac{2 \eta_{H_{\|}}}{\eta_{H_{\perp}}}=\frac{\eta_{\|}}{\eta_{\perp}}=\frac{\sigma_{\|}}{\sigma_{\perp}}=\left.\frac{f}{h}\right|_{r=r_{0}},
$$

where $\sigma_{\|}=\sigma_{z z}=\left.(f / \sqrt{h})\right|_{r=r_{0}}, \sigma_{\perp}=\sigma_{x x}=\sigma_{y y}=\left.\sqrt{h}\right|_{r=r_{0}}$.

Finally, let us comment on the temperature scaling behavior of viscosities and conductivities in the quantum critical regime. At zero temperature, there is an emergent Lifshitz-like symmetry in the IR at the transition point $M / b \simeq 0.744$ and $\mathrm{IR}$ physics is invariant under $\left(t, x, y, r^{-1}\right) \rightarrow l\left(t, x, y, r^{-1}\right), z \rightarrow l^{\beta} z$ with the anisotropic scaling exponent $\beta \simeq 0.407$ together with $f \rightarrow l^{-2} f$, $h \rightarrow l^{-2 \beta} h, A_{z} \rightarrow l^{-\beta} A_{z}, \phi \rightarrow \phi$, [14]. At very low temperature, since $T \rightarrow l^{-1} T$ we can obtain the temperature

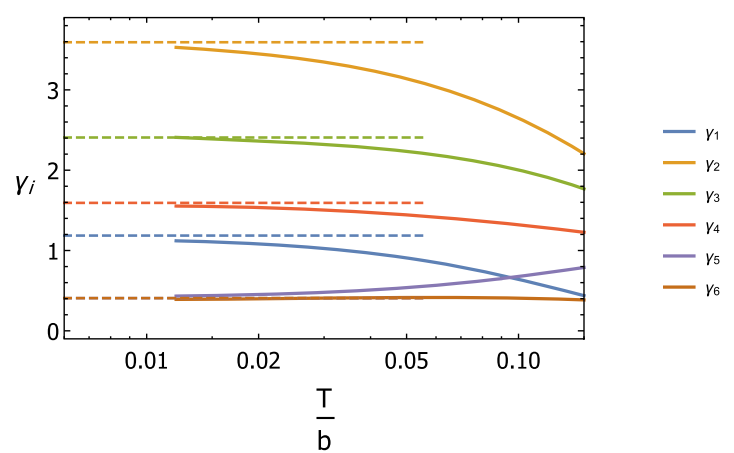

FIG. 4. The temperature scaling exponents $\gamma_{i}$ with $i \in$ $\{1, \ldots, 6\}$ for viscosities $\eta_{\|}, \eta_{H_{\|}}$, and $\eta_{H_{\perp}}$ and for electric conductivities $\sigma_{\|}, \sigma_{\perp}$, and $\sigma_{\mathrm{AHE}}$ at the critical value $M / b=$ 0.744 for low temperatures. The dashed lines in the picture denote the analytic values of the scaling exponents from the scaling analysis.

scaling behavior of the viscosities and conductivities near the critical region from scaling arguments. More precisely, at the critical regime, when $M / b \rightarrow 0.744$, we have $\eta_{\|} / s \propto T^{\gamma_{1}}, \quad \eta_{H_{\|}} \propto T^{\gamma_{2}}, \quad \eta_{H_{\perp}} \propto T^{\gamma_{3}}$ with $\left(\gamma_{1}, \gamma_{2}, \gamma_{3}\right)=$ $(2-2 \beta, 4-\beta, 2+\beta)$ and $\sigma_{\|} \propto T^{\gamma_{4}}, \sigma_{\perp} \propto T^{\gamma_{5}}, \sigma_{\mathrm{AHE}} \propto$ $T^{\gamma_{6}}$ with $\left(\gamma_{4}, \gamma_{5}, \gamma_{6}\right)=(2-\beta, \beta, \beta)$ for low temperatures [47]. In Fig. 4 we plot the temperature scaling exponents $\gamma_{i}$ with $i \in\{1, \ldots, 6\}$ of our numerical results at low temperature at the critical value of $M / b$. We can see from the figure that the scaling exponents are approaching the analytic values when the temperature decreases. These scaling dependences explain the peak and dip behavior of the transports of holographic Weyl semimetal in the critical regime.

While our results have been obtained using a holographic model we do expect that the lessons one can draw from it have much wider applicability. We strongly believe that the presence of odd viscosities is a universal feature of the quantum critical region of Weyl semimetal-semimetal transitions. At weak coupling this is the region which is characterized by quadratic band touching in one of the directions in momentum space (the $z$ direction of our model). Our holographic model gives therefore an exciting new prediction for the physics of the quantum critical region of Weyl semimetals.

We thank R. G. Cai, A. Cortijo, Y. Ferreiro, F. D. M. Haldane, E. Megias, S. Sachdev, G. W. Semenoff, M. A. H. Vozmediano, Q. Yang, and J. Zaanen for useful discussions. This work has been supported by Projects No. FPA2012-32828, No. FPA2015-65480-P, and by the Centro de Excelencia Severo Ochoa Programme under Grant No. SEV-2012-0249. Y. L. would like to acknowledge support by the Thousand Young Talents Program of China. The work of Y.W.S. was also supported by the European Union through a Marie Curie Individual Fellowship No. MSCA-IF-2014-659135. 
*karl.landsteiner@csic.es

†yanliu.th@gmail.com *yawen.sun@csic.es

[1] J. Zaanen, Y. W. Sun, Y. Liu, and K. Schalm, Holographic Duality in Condensed Matter Physics (Cambridge University Press, Cambridge, 2015); M. Ammon and J. Erdmenger, Gauge/Gravity Duality: Foundations and Applications (Cambridge University Press, Cambridge, 2015); H. Nastase, Introduction to the AdS/CFT Correspondence (Cambridge University Press, Cambridge, 2015).

[2] J. Zaanen, Science 351, 1026 (2016).

[3] D. A. Bandurin et al., Science 351, 1055 (2016).

[4] J. Crossno et al. Science 351, 1058 (2016).

[5] P. J. W. Moll, P. Kushwaha, N. Nandi, B. Schmidt, and A. P. Mackenzie, Science 351, 1061 (2016).

[6] S.-Y. Xu et al., Science 349, 613 (2015).

[7] B. Q. Lv et al., Phys. Rev. X 5, 031013 (2015).

[8] L. Lu, Z. Wang, D. Ye, L. Ran, L. Fu, J. D. Joannopoulos, and M. Soljacic, Science 349, 622 (2015).

[9] A. Vishwanat, Phys. Rev. X 5, 031013 (2015).

[10] O. Vafek and A. Vishwanat, Annu. Rev. Condens. Matter Phys. 5, 83 (2014).

[11] P. Hosur and X. Qi, C.R. Phys. 14, 857 (2013).

[12] J. Casalderrey-Solana, H. Liu, D. Mateos, K. Rajagopal, and U. A. Wiedemann, arXiv:1101.0618.

[13] K. Landsteiner and Y. Liu, Phys. Lett. B 753, 453 (2016).

[14] K. Landsteiner, Y. Liu, and Y. W. Sun, Phys. Rev. Lett. 116, 081602 (2016).

[15] Here $L$ is the scale of the AdS space. In the following, we set $2 \kappa^{2}=e^{2}=L=1$.

[16] The same setup with different boundary conditions was also used in $[17,18]$ to realize axial charge dissipations in the study of negative magnetoresistivity of the holographic Dirac semimetal.

[17] A. Jimenez-Alba, K. Landsteiner, Y. Liu, and Y. W. Sun, J. High Energy Phys. 07 (2015) 117.

[18] Y. W. Sun and Q. Yang, arXiv:1603.02624.

[19] A. A. Burkov and L. Balents, Phys. Rev. Lett. 107, 127205 (2011).

[20] A. G. Grushin, Phys. Rev. D 86, 045001 (2012).

[21] A. A. Zyuzin and A. A. Burkov, Phys. Rev. B 86, 115133 (2012).

[22] G. E. Volovik, arXiv:1604.00849.

[23] S. Sachdev, Quantum Phase Transitions (Cambridge University Press, Cambridge, 1999).

[24] J. E. Avron, R. Seiler, and P. G. Zograf, Phys. Rev. Lett. 75, 697 (1995).

[25] C. Hoyos, Int. J. Mod. Phys. B 28, 1430007 (2014).

[26] E. M. Lifschityz and L.P. Pitaevski, Physical Kinetics (Butterworth-Heinemann, Cambridge, 1981).
[27] F. M. Haehl, R. Loganayagam, and M. Rangamani, J. High Energy Phys. 05 (2015) 060.

[28] K. Landsteiner, E. Megias, and F. Pena-Benitez, Phys. Rev. Lett. 107, 021601 (2011).

[29] K. Landsteiner, E. Megias, L. Melgar, and F. Pena-Benitez, J. High Energy Phys. 09 (2011) 121.

[30] S. Golkar and D. T. Son, J. High Energy Phys. 02 (2015) 169.

[31] K. Jensen, R. Loganayagam, and A. Yarom, J. High Energy Phys. 02 (2013) 088.

[32] In a $3+1$ dimensional axisymmetric system with a time reversal breaking vector, there are seven components in the viscosity tensor, including three shear viscosities, two odd viscosities, and two bulk viscosities. Besides the four components below, there are another two bulk viscosities and one shear viscosity which come from the spin zero components of $x x+y y$ and $z z$, which we do not consider in this Letter.

[33] A. Cortijo, Y. Ferreiros, K. Landsteiner, and M. A. H. Vozmediano, Phys. Rev. Lett. 115, 177202 (2015).

[34] O. Saremi and D. T. Son, J. High Energy Phys. 04 (2012) 091.

[35] J. W. Chen, S. H. Dai, N. E. Lee, and D. Maity, J. High Energy Phys. 09 (2012) 096.

[36] R. G. Cai, T. J. Li, Y. H. Qi, and Y. L. Zhang, Phys. Rev. D 86, 086008 (2012).

[37] D. C. Zou, Y. Liu, and B. Wang, Phys. Rev. D 89, 064036 (2014).

[38] H. Liu, H. Ooguri, and B. Stoica, Phys. Rev. D 90, 086007 (2014).

[39] W. Fischler and S. Kundu, J. High Energy Phys. 04 (2016) 112.

[40] T. Y. Zhao and T. Wang, J. Cosmol. Astropart. Phys. 06 (2016) 019.

[41] See Supplemental Material at http://link.aps.org/ supplemental/10.1103/PhysRevLett.117.081604 for details.

[42] See Supplemental Material at http://link.aps.org/ supplemental/10.1103/PhysRevLett.117.081604 for details on equations, solutions, and figures supporting statements on the dissipative shear viscosities made in the Letter, which includes Refs. [43-46].

[43] P. K. Kovtun, D. T. Son, and A. O. Starinets, Phys. Rev. Lett. 94, 111601 (2005).

[44] A. Rebhan and D. Steineder, Phys. Rev. Lett. 108, 021601 (2012).

[45] K. A. Mamo, J. High Energy Phys. 10 (2012) 070.

[46] S. Jain, N. Kundu, K. Sen, A. Sinha, and S. P. Trivedi, J. High Energy Phys. 01 (2015) 005.

[47] We emphasize that the scaling exponent $\beta$ depends on the free parameters in the holographic model, while the scaling behavior in the critical regime is parameter independent and universal. 